\title{
Kanonische Texte in poetischer Form
}

\section{Zur Versifikation von Evangelienabschnitten im Berliner Hans Sachs-Autograph}

Bekanntlich steht ab der Reformation die Versifikation ausgewählter Bibeltexte im Zentrum des Meistergesangs. In bislang ungezählter Anzahl wurden die Bücher der beiden Bibeln vom Buch Genesis bis zur Apokalypse ausschnittweise, in der Addition der bearbeiteten Abschnitte teilweise sogar mehr oder minder vollständig, in ausgewählten Episoden (etwa die Geburtsgeschichte Jesu nach dem Lukas-Evangelium) auch mehr- und vielfach in die Form von Meisterliedern gegossen. ${ }^{1}$ Durch diese Häufung entstand ein eigener Liedtyp der textnahen Bibelversifikation, der dem zu Beginn der Reformationszeit um 1520 merklich lahmenden Meistergesang $\mathrm{zu}$ neuer Lebenskraft verhalf. Die entscheidende Weichenstellung zu diesem Aufschwung erfolgte durch Hans Sachs, der nach seiner Wendung zur Reformation ${ }^{2}$ ab 1526 als erster Meistersinger des neuen Zeitalters mit Bibelversifikationen in Meistertönen begann. ${ }^{3}$ Auslöser und Grundlage für diese Neuausrichtung war Martin Luthers Bibelübersetzung (ab 1522), die dem Meistergesang mit dem Liedtyp der textnahen Bibelversifikation als Instrument der volkssprachlichen, auf dem Bibeltext basierenden Glaubensverkündigung zu neuer, langdauernder Legitimation verhalf. ${ }^{4}$

Diesen eindeutigen Sachverhalt können auch die Bibelversifikationen nicht in Frage stellen, die von Sangspruchdichtern und vorreformatorischen Meistersingern überliefert sind. Dagegen sprechen bereits die Zahlenverhältnisse: Seit

1 Vgl. dazu das Verzeichnis der Bibelstellen in: RSM 16, S. 261-330. Die textnahen Versifikationen (aus dem älteren und jüngeren Teil des Repertoriums) sind mit einem Asteriskus ${ }^{\star}$ ) gekennzeichnet. Die Liedsiglen entsprechen nachfolgend dem Repertorium.

21523 legte Hans Sachs mit seinem Meisterlied ,Die Nachtigall‘ und besonders mit dem im selben Jahr gedruckten Spruchgedicht, Die Wittenbergisch Nachtigall‘ ein offenes Bekenntnis zu Luthers Lehre ab.

3 Im selben Jahr veröffentlichte Hans Sachs eine Flugschrift mit 13 Psalmliedern, denen kein Meisterton zugrunde liegt. Sie dürften von Martin Luthers sieben Psalmliedern angeregt worden sein, die mit einer Ausnahme (1526) 1523/24 gedruckt vorlagen. In Luthers Spuren wandelte Sachs zuvor schon, als er 1525 acht Kirchenlieder (Etliche geystliche in der schrifft [,Bibel'] gegrünte [,gegründete'] lieder für die layen zu singen) als Flugschrift bei Jobst Gutknecht in Nürnberg, dem Drucker des ,Achtliederbuchs', herausbrachte. Darüber hinaus wurde Hans Sachs als Kirchenlieddichter nicht mehr tätig, sondern wandte sich ab 1526 wieder dem Meistergesang zu. Vgl. dazu Brunner: ...das Reich, S. 336-349, zu den Psalmliedern S. 340 - 342 und Brunner: Luther, S. 255 f. 4 Vgl. dazu insgesamt Brunner: Meistergesang.

Ә OpenAccess. ( 2020 Johannes Janota, publiziert von De Gruyter. (cc) BY Dieses Werk ist lizenziert unter der Creative Commons Attribution 4.0 International. https://doi.org/10.1515/9783110666816-011 
dem dreizehnten Jahrhundert sind nur an die 90 Versifikationen bekannt, von denen zudem knapp 70 auf Michel Beheim, den letzten professionellen Spruchsänger, entfallen, der trotz seines imposanten Euvres ohne erkennbaren Einfluss auf den Meistergesang blieb. Lediglich 23 Versifikationen anderer Dichter bilden ein kaum wahrnehmbares Rinnsal, das zum breiten Strom der Sangsprüche und frühen Meisterlieder nicht nennenswert beiträgt. Zurecht stellte daher Burghart Wachinger fest, dass es die Bibelversifikation als festen Liedtyp erst seit der Reformation gibt. ${ }^{5}$

Dennoch zeigte bereits der frühe Hans Sachs thematisches Interesse an Bibelversifikationen, der in seiner ersten autographen Meisterliedersammlung von 1517/18, dem Berliner Autograph (Berlin, SBB, mgq 414), ${ }^{6}$ nicht weniger als zehn Texte dieser Art aufgezeichnet hat, die vom RSM als textnahe eingestuft werden und die noch nicht unter reformatorischem Einfluss stehen. ${ }^{7}$ Von den 23 vorreformatorischen Versifikationen wird somit annähernd die Hälfte (nämlich 43,5\%) durch Hans Sachs überliefert. Dahinter steht vielleicht eine Vorliebe der Nürnberger Meistersinger, denn mit den Ton- bzw. Textautoren Konrad Nachtigall, Hans Folz und Lienhard Nunnenbeck (der Sachs im Meistergesang unterrichtete) führen fünf der zehn Versifikationen im Sachs-Autograph unmittelbar nach Nürnberg. ${ }^{8}$ Allerdings hat Sachs nicht gezielt nach Bibelversifikationen gefahndet, vielmehr sind sie Teil der verschiedenen Autor- und Tönesammlungen (Nunnenbeck, Regenbogen, Marner, Folz) innerhalb seines Autographs. Dennoch lässt sich fragen, ob Hans Sachs bereits vor der Reformation von den Meistersingern seiner Heimatstadt, aber auch von der ihm darüber hinaus zugänglichen Sangspruch- und frühen Meisterliedtradition Anregungen für die eigenen Bibelversifikationen als Ausdruck seines Bekenntnisses zur Reformation erhalten hat. Dabei kann die kritische Auseinandersetzung mit dem Repertoire seiner Sammlung von 1517/18 bei Hans Sachs zu einer Anlehnung an tradierte Muster wie zur Ablehnung bestimmter Ausprägungen geführt haben. Auf jeden Fall vermitteln aber die Aufzeichnungen im Sachs-Autograph einen differenzierten Einblick in

5 Vgl. Wachinger: Michel Beheim. Zitiert nach dem ergänzten Wiederabdruck in Wachinger: Lieder, S. 363-393, hierzu S. 387: „Als festen Typ gibt es die Bibelversifikation außer bei Beheim nur im nachreformatorischen Meistergesang, wo die Lutherbibel zur verbindlichen Grundlage der reinen Lehre geworden war und beim Liedvortrag von den Merkern hinterm Vorhang zur Kontrolle mitgelesen wurde.“

6 Vgl. RSM 1, S. 84-86.

7 Gleichzeitig dokumentiert diese Sammlung, die Sachs mit eigenen Liedern eröffnete, dass er als Autor in diesem Bereich vor der Reformation nicht produktiv wurde.

8 Für die 5 anderen Lieder lässt sich keine Nürnberger Provenienz sichern. Insgesamt hat Hans Sachs in seinem Autograph folgende zehn Bibelversifikationen aufgezeichnet: ${ }^{1}$ Marn $/ 7 / 572,{ }^{1}$ Frau/ 23/6b, ${ }^{1}$ Regb/4/651-653, ${ }^{1}$ NachtK/8/1, ${ }^{1}$ Folz/33b und 132, ${ }^{1}$ Nun/20 und 26. 
die unterschiedlichen Gestaltungsweisen und in die Spezifik der vorreformatorischen Bibelversifikationen, die dann den reformatorischen Zuschnitt der Versifikationen genauer erkennen lassen, die Hans Sachs zwischen 1526 und 1567 vorgelegt hat. ${ }^{9}$

Da sich Sachs bei seinen Versifikationen um eine besondere Nähe zur LutherÜbersetzung als seiner Textvorlage bemüht, muss der Aspekt der Textnähe ein leitendes Kriterium bei der Analyse der zehn Aufzeichnungen im frühen SachsAutograph sein. Sollten sich nämlich Unterschiede in der intendierten Textnähe ${ }^{10}$ ergeben, dann läge hier ein aufschlussreiches Merkmal bei der Unterscheidung zwischen vor- und nachreformatorischen Versifikationen vor. Im Zusammenhang mit Bibelversifikationen unterliegt der Begriff der Textnähe allerdings deutlichen Einschränkungen. Darauf weist bereits das Lexem ,-nahe“ in der Kennzeichnung „textnahe“, mit der das RSM stillschweigend operiert. ${ }^{11}$ Es stellt in Rechnung, dass eine wortgetreue Wiedergabe des Bibeltextes im Rahmen komplizierter Strophenformen unmöglich ist. Angesichts der formalen Herausforderungen der Meisterliedtöne muss man hier größere Lizenzen bei syntaktischen Veränderungen, bei Füllwörtern und -versen einräumen, wenn der Begriff der Textnähe nutzbringend gebraucht werden soll. Von diesen weitgehend bedeutungsneutralen Lizenzen abzugrenzen sind die Fälle, die nicht auf eine textnahe, sondern bloß paraphrasierende Versifikation zielen, die in eine bibelnahe Versifikation erklärende oder deutende Zusätze inserieren, die den versifizierten Bibeltext mit Autortexten rahmen u.a.m. Diese Fälle, bei denen die Versifikation nach Art der Predigt- und der Traktatliteratur oder der Plenarien mit einem deutenden Netz überzogen sind, erfüllen - anders als vom RSM gesehen - nicht das Kriterium der Textnähe, die nur für solche Versifikationen gelten soll, die sich von deutenden und das Verstehen lenkenden Autortexten (weitgehend) freihalten oder aber beide Ebenen strikt trennen. Diese Abgrenzung versteht sich nicht als Werturteil über das Unvermögen, eine Bibelstelle textnahe $\mathrm{zu}$ versifizieren, sondern als Hinweise auf andere Textfunktionen der Versifikation.

9 Die erste im RSM gebuchte Versifikation ( $\left.{ }^{2} \mathrm{~S} / 111\right)$ stammt von 1526, die letzte ( $\left.{ }^{2} \mathrm{~S} / 6091 \mathrm{~A}\right)$ ist auf den 08.12.1567 datiert.

10 Bei den vorreformatorischen Versifikationen kann allerdings nur allgemein auf die Vulgata zurückgegriffen werden, weil wir - außer vereinzelt bei Michel Beheim - bislang keine konkreten Vorlagen kennen. Man wird dazu vor allem die vielen noch unerschlossenen Plenarien wie die nach wie vor schwer überschaubaren Predigt- und Traktatliteratur gezielt durchsuchen müssen. Zu den Plenarien vgl. Reinitzer, Schwencke, Sp. 737-763 und den Art. ,Plenarien [Korr./Nachtr.]‘. In: VL 11 (2004), Sp. 1249 (Nachträge und weitere Verweise).

11 RSM 16, S. 261. 
Innerhalb des Berliner Autographs verhindert bei zwei siebenstrophigen Versifikationen bereits der Typ des Glossenliedes den Anspruch auf Textnähe. ${ }^{12}$ Die beiden Anonyma ( ${ }^{1}$ Marn/7/572 und ${ }^{1}$ Regb/4/651) zitieren wortweise in Latein den Prolog des Johannes-Evangeliums (Joh 1,1-14) und paraphrasieren oder kommentieren ihn auf Deutsch. Das Verfahren zeigt bereits der erste Stollen des Aufgesangs in ${ }^{1}$ Marn/7/572 (V. 1-6; Hervorhebungen hier und in den nachfolgenden Textzitaten von mir):

\footnotetext{
Jn principio sing ich hi

erat verbum da pey

im anefang lob wort was ye

pey Got hoch in der jerarchey

et verbum erat wol betracht

aput [D]eum mit suessem hal
}

In vorreformatorischer Zeit schuf dieser Liedtyp die Möglichkeit, den approbierten lateinischen Bibeltext mit einer deutschsprachigen Versifikation zu kombinieren. Dieses Verfahren wurde aber hinfällig, als Luthers Bibelübersetzung den Rang eines gültigen Textes einnahm. Es diente auch Hans Sachs nicht als Muster für seine Bibelversifikationen. Dahinter scheint eine bewusste Entscheidung zu stehen, denn er war mit dem Typ des lateinisch-deutschen Glossenlieds nicht nur vertraut, sondern verwendete ihn vor seinem Bekenntnis zur Reformation auch für eigene Lieder. ${ }^{13}$

Einen anderen Weg beschritt Lienhard Nunnenbeck (gest. vor 1527) bei seiner Versifikation des Johannes-Prologs. Er inserierte beim fünfstrophigen Lied ${ }^{1} \mathrm{Nun} /$ $20^{14}$ in die Versifikation der schwerverständlichen Perikope kontinuierlich erläuternde Kommentare. Das veranschaulicht der Abgesang der ersten Strophe, mit dem die Versifikation einsetzt ${ }^{15}$ (V. 13-27):

12 Bei einer weiteren Glossierung des Johannes-Prologs im mgq 414 ('Zorn/4/22) sieht auch das RSM keine Textnähe bei der Versifikation.

13 Im Berliner Autograph hat Hans Sachs nicht weniger als sieben Glossenlieder anderer Autoren aufgezeichnet. Es handelt sich dabei um Glossierungen des Gebets ,Ave Maria، (vier Lieder: ${ }^{1} \mathrm{Frau} / 22 / 2 \mathrm{~b},{ }^{1} \mathrm{Nun} / 24$ und 31, ${ }^{1} \mathrm{Zorn} / 3 / 10$ ), des Athanasianischen Glaubensbekenntnisses (ein Lied: ${ }^{1} \operatorname{Regb} / 4 / 657$ ), des ,Salve Regina' (zwei Lieder: ${ }^{1}$ Barz/1, $\left.{ }^{1} \mathrm{Sing} / 2 / 2\right)$. Von sich selbst hat er 4 Glossenlieder im mgq 414 aufgezeichnet: zum Hymnus ,Ave maris stella (ein Lied: ${ }^{2} \mathrm{~S} / 44 \mathrm{~b}$ ), zur Doxologie ,Gloria patri' (ein Lied: ${ }^{2} \mathrm{~S} / 25 \mathrm{~b}$ ) und zur Antiphon ,Salve Regina' (zwei Lieder: ${ }^{2} \mathrm{~S} / 29 \mathrm{~b}$ und 44b).

14 Vgl. die Ausgabe von Klesatschke, S. 299-303 (Edition), S. 407-409 (Kommentar).

15 Der Aufgesang besteht aus einem Autortext, in dem Nunnenbeck den Evangelisten Johannes (erster Stollen) und den Heiligen Geist (zweiter Stollen) um Beistand anruft. 


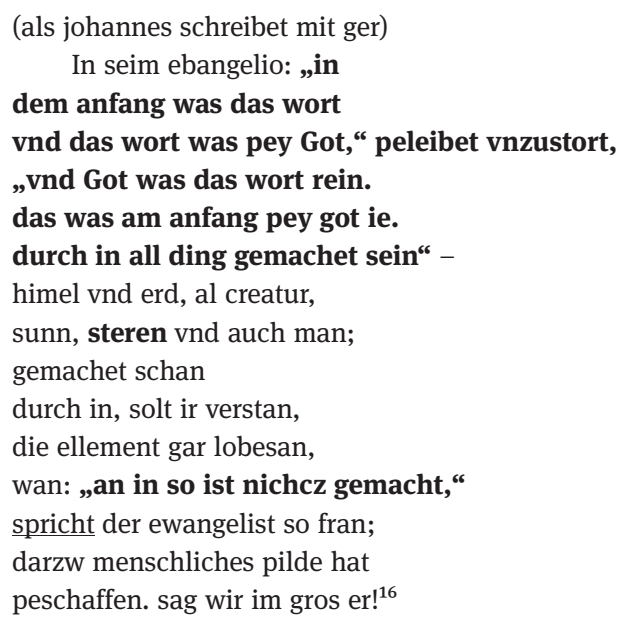

Nunnenbeck folgte damit einem Muster, das aus der Predigt, aus der Traktat- und Erbauungsliteratur bestens bekannt ist. Im Liedvortrag wird dieses Verfahren allerdings zum Problem, weil man als Hörender - sieht man von den gelegentlichen Markierungen durch Inquit-Formeln ab (etwa V. 25) - nicht zwischen Bibelund Autortext unterscheiden kann. Deswegen fällt es schwer, hier von einer textnahen Bibelversifikation zu sprechen, obwohl es Nunnenbeck gelingt, den anspruchsvollen Text ziemlich getreu zu versifizieren. Darin unterscheidet sich seine zweite Bibelversifikation im dreistrophigen Lied ${ }^{1} \mathrm{Nun} / 26^{17}$, der das Evangelium (Lk 1,26-38) zum Fest Mariä Verkündigung (25. März) zugrunde liegt. Durch Raffungen und zitathafte Textauswahl wird das Geschehen so stark kondensiert, dass es in zwei 20zeilige Strophen passt ${ }^{18}$ und sogar noch Platz bleibt für eine etwas kühne Erklärung der jungfräulichen Empfängnis (II,15-18):

sich schwang der himelhortte

wol vnder iren geren [,Kleidersaum', auch ,Schoß'],

do ward schwanger die iunckfraw clar

aus wirckvng gotlicher volleist.

Innerhalb der Grenzen, die bei den beiden Liedern Nunnenbecks sichtbar wurden, bewegen sich - mit je eigenen Facetten - zwei weitere anonyme Bibelver-

16 Vgl. dazu Joh 1,1-3: ${ }^{1}$ In principio erat verbum, et verbum erat apud Deum, et Deus erat verbum. ${ }^{2}$ Hoc erat in principio apud Deum. ${ }^{3}$ Omnia per ipsum facta sunt, et sine ipso factum est nihil, quod factum est.

17 Vgl. Klesatschke: Nunnenbeck (Anm. 14); S. 138-140 (Edition), S. 357f. (Kommentar).

18 Die dritte Strophe ist reiner Autortext, der sich an Maria wendet. 
sifikationen im Berliner Sachs-Autograph. Das eine Meisterlied ('Frau/23/6b) greift erneut den Johannes-Prolog auf, der in Ausschnitten paraphrasiert wird, um daraus in der (3.) Schlussstrophe eine Mahnung zum würdigen Empfang der Eucharistie abzuleiten. In der älteren Fassung der ,Kolmarer Liederhandschrift" $\left({ }^{1} \mathrm{Frau} / 23 / 6 \mathrm{a}\right){ }^{19}$ wurde dagegen die Menschwerdung des Gottessohnes durch Maria thematisiert. Die beiden unterschiedlichen Schlüsse zeigen anschaulich, dass es bei der Versifikation nicht um Textnähe ging, sondern um eine biblische Grundlegung der theologischen Thematik in der Schlussstrophe beider Fassungen. Damit rückt der Bibeltext aus dem Zentrum, ihm kommt lediglich eine dienende Funktion zu.

Das andere Meisterlied $\left({ }^{1} \mathrm{NachtK} / 8 / 1\right)^{20}$ versifiziert trotz artifizieller Strophenform mit Schlag- und Tiradenreimen das Geschlechterregister des MatthäusEvangeliums (Mt 1,1-16) in fünf Strophen erstaunlich textnahe (was durch die syntaktische Struktur des Registers allerdings auch erleichtert wurde). Das belegt etwa der erste Stollen der zweiten Strophe (II,1-7):

\footnotetext{
Jüdas Fares gepare

und Sare von der schün Thomar.

Vares gepar vürbare

Esram an des geschlechtes schar.

Esram gepar Aram; Aras sant sein sün dar.

von in ist poren woren

Aminadab der here. ${ }^{21}$
}

Trotz dieser Textnähe zielte der anonyme Autor aber auf keine durchgehende Wiedergabe der Perikope, sondern rahmte und durchsetzte wie Nunnenbeck die Bibelversifikation mit Autortexten, deren Lichtmotivik mit der Schlussstrophe in einen Marienpreis münden. Sieht man von den Glossenliedern ab, so ist diese Mischung von Bibel- und Autortext die - vielfach variierte - Normalform bei den vorreformatorischen liedhaften Bibelversifikationen.

Scheinbar folgt auch Hans Folz mit seiner siebenstrophigen Versifikation des Johannes-Prologs ( $\left.{ }^{1} \mathrm{Folz} / 33 \mathrm{~b}\right)^{22}$ dieser Tradition, weil hier wiederum Bibel- und Autortext kombiniert sind. Bei genauerem Hinsehen zeigt sich jedoch, dass im

19 Abdruck bei Baldzuhn, S. 213f., mit dem veränderten Schluss der Berliner Handschrift (S. 214f.); zu den beiden unterschiedlichen Schlussstrophen vgl. S. $215 \mathrm{f}$.

20 Vgl. den Abdruck von Cramer, S. 215-218 (Text) und S. 411 (metrisches Schema).

21 Vgl. Mt 1,3 f.: ${ }^{3}$ Iudas autem genuit Phares et Zara de Thamar. Phares autem genuit Esrom. Esrom autem genuit Aram. ${ }^{4}$ Aram autem genuit Aminadab.

22 Das Meisterlied wird von Folz zuvor schon in einer autographen Sammlung München, BSB, Cgm 6353 von 1480/90 überliefert ( ${ }^{1}$ Folz/33a). Vgl. die Edition von Mayer, S. 136-141. 
vorliegenden Meisterlied die beiden Ebenen säuberlich getrennt sind. Der erste Stollen einer jeden Strophe versifiziert sehr textnahe einen Abschnitt der Perikope, der anschließend - gerade bei diesem schwierigen Text didaktisch sehr geschickt - im zweiten Stollen und im Abgesang erklärt und theologisch gedeutet wird. Das Verfahren illustriert ein Ausschnitt aus der ersten Strophe (V. 1-19):

In dem anfang so was das worte, und das wort was pey Got, und Got was das wort ye, und das was im anfang pey Got, und alle ding sint durch das wort gemachet.

Syn, mensch, was her in sey der horte [,Bedeutung'] : der sun was ye im vater sein, vermercket wye der vater ye im sun sich hot, mit yn der geist ein wesen ungesachet.

Wan allß kein vater on ein sun, sun on ein vater auch nie wart erkunde, $[\ldots]$

Auf solche Weise erhält man durch eine kontinuierliche Lektüre der ersten Stollen eine vollständige Wiedergabe des Johannes-Prologs. Diese säuberliche Trennung der beiden Textebenen zeigt, dass es neben deren üblichen Vermengung nicht nur ein Wissen um die übergeordnete Autorität des Bibeltextes gab, sondern auch eine genaue Vorstellung von seiner inhaltlichen Unantastbarkeit, die durch inserierte Autorkommentare, durch Raffungen oder durch zitathafte Auswahl bei einer Bibelversifikation verletzt wurde. Das belegt auch ein dreistrophiges Lied ( ${ }^{1} \mathrm{Regb} / 4 /$ 633) in der ,Heidelberger Meisterliederhandschrift" (UB, Cpg 392), ${ }^{23}$ das bei der gleichfalls textnahen Versifikation der Versuchung Jesu (Mt 3,1-11) zwar nicht wie Folz formal die beiden Textebenen trennt, wohl aber durch eine sprachliche Markierung mit der Publikumsanrede mensch an der Stelle des Abgesangs, an der in jeder Strophe der Autorkommentar einsetzt. Als Beispiel sei der Beginn des Abgesangs in der zweiten Strophe zitiert (V. 9-12):

Jhesus im da sein antwurt gab:

got deinem herren solt du nit versuchen.

10

o mensch dabei ein urkund hab,

das du dich in der zeit nit solt entruchen [,nicht kümmern'].

23 Digitalisat der Handschrift unter: http://digi.ub.uni-heidelberg.de/diglit/cpg392 (09. Oktober 2019). 
Wenn Hans Sachs sich dennoch nicht diesem Modell anschloss, als er mit seinen Bibelversifikationen begann, dann liegt das wohl daran, dass sich die versifizierten Bibelverse im Folz-Lied über alle sieben Strophen verteilen und sie so den Eindruck von separierten Zitaten vermitteln, die jeweils nur im Dienst der anschließenden Deutungen stehen. Im Blick auf die vorlagengetreue Versifikation im jeweils ersten Stollen wird man diesem Meisterlied, das Sachs in sein Autograph aufnahm, den Rang einer textnahen Bibelversifikation jedoch nicht streitig machen dürfen.

Eine andere Möglichkeit für die Trennung von Bibel- und Autortext führt ein siebenstrophiges Lied ( ${ }^{1}$ Folz/132) vor, ${ }^{24}$ das Folz nicht sicher zugeordnet werden kann, weil es nur im Sachs-Autograph überliefert wird und damit außerhalb der Korpusüberlieferung steht. Hier rahmen Autorkommentare die insgesamt textnahe Versifikation der Auferstehung Jesu nach dem Bericht bei Matthäus (Mt 28,1-19). Ein Zitat aus dem Abgesang der ersten Strophe, in dem die Versifikation einsetzt, ${ }^{25}$ illustriert dieses Verfahren (V. 14-21):

da von sagt das ewangely

secundum Mateum, merckt wy:

nach des sabacz abent geschach,

e das auf prach

des dages morgenröte,

Maria Magdalena frw

und auch dy zwu

den herren eilten zw salben in nete [,mit Eifer'].

Die Grenze zwischen den beiden Textebenen markieren hier der Quellennachweis und die Publikumsanrede. (I,14f.). ${ }^{26}$ Dabei deutet die Formulierung ewangely secundum Mateum auf einen gottesdienstlichen Hintergrund hin. Tatsächlich wurde hier das Evangelium der ersten Ostermesse nach dem Abschluss der Auferstehungsfeier versifiziert. Diese postulierte Verbindung mit der Liturgie erscheint etwas gekünstelt zu sein, sie findet aber eine Stütze in der Versifikation, die (V. 20f.) abweichend von Matthäus nicht zwei, sondern wie bei Markus drei Frauen nennt, die - wie ebenfalls bei Markus (16,1) - den Leichnam Jesu salben

24 Vgl. die Ausgabe von Mayer, S. 311-314.

25 Mt 28,1: Vespere autem sabbati, quae lucescit in prima sabbati, venit Maria Magdalena et altera Maria videre sepulchrum.

26 Das gilt auch für die drei abschließenden Strophen, die auf den sechsfachen Nutzen der Auferstehung (V) und auf die fünf Wundmale Jesu (VII) hinweisen, um dann zu Dank und Lob der Erlösungstat Jesu aufzufordern (VII). Hier markiert das ich den Übergang zum Autortext: Sechs nucz vünd ich der urstent hy $(\mathrm{V}, 1)$. 
wollten (bei Matthäus: um das Grab aufzusuchen). ${ }^{27}$ Weitere Abweichungen von der Perikope finden sich im Abgesang der zweiten Strophe (V. 16-21):

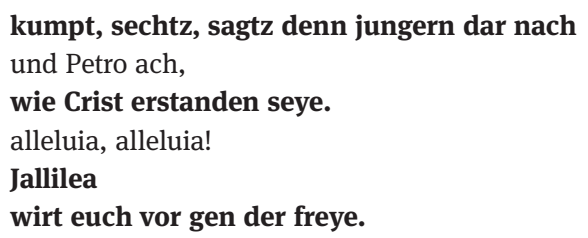

wirt euch vor gen der freye.

Die Engelbotschaft an die Frauen am Grab folgt zwar wieder dem MatthäusEvangelium, ${ }^{28}$ die Nennung des Petrus wird allerdings erneut dem MarkusEvangelium entnommen. ${ }^{29}$ Nichtbiblisch ist der doppelte Alleluia-Ruf, der Teil der Antiphon Venite et videte locum (II,16: kumpt, sechtz) in einer lateinischen Osterfeier des Typs II ist. ${ }^{30}$ Danach schwenkt der Autor wieder in die (trotz einiger Raffungen insgesamt textnahe) Versifikation der Perikope ein. Die Wahl des Bibelabschnitts für die Versifikation scheint also nicht willkürlich erfolgt zu sein, sondern hatte zumindest in Einzelfällen einen gottesdienstlichen (hier: die dramatisierte Osterfeier im Anschluss an die Matutin) oder liturgischen Hintergrund (hier: Evangelium der ersten Ostermesse). Hierzu stellt sich auch ein Hinweis in der Schlussstrophe (V) der bereits genannten Versifikation des Johannes-Prologs durch Nunnenbeck ( $\left.{ }^{1} \mathrm{Nun} / 20\right)$, der zu Beginn des Abgesangs daran erinnert, dass diese Perikope im Anschluss an die Messe vom Priester halblaut rezitiert wurde (V. 13-16):

Das ewangely ist der grundt, darauf der gelaub stat.

der priester sprichet es, wan er gelessen hat

Sein mes, solt ir verstan. ${ }^{31}$

Die Anbindung eines Meisterliedes an kirchliche Festtage und deren Perikopen wird bei den zwei letzten Belegen im Sachs-Autograph explizit genannt. Dort folgen die beiden dreistrophigen Lieder ${ }^{1}$ Regb/4/652 und ${ }^{1} \operatorname{Regb} / 4 / 653$ zudem unmittelbar aufeinander. Das Lied ${ }^{1}$ Regb/4/652, das einen Abschnitt aus dem Hohen

27 Vgl. Mk 16,1: Et cum transisset sabbatum, Maria Magdalena et Maria Iacobi et Salome emerunt aromata, ut venientes ungerent Iesum. (Vgl. auch Lk 24,1 und 10.)

28 Vgl. Mt 28,6f.: Non est hic. Surrexit enim, sicut dixit. Venite et videte locum, ubi positus erat Dominus. ${ }^{7}$ Et cito euntes dicite discipulis eius, quia surrexit. Et ecce praecedit vos in Gallileam. 29 Vgl. Mk 16,7: Sed ite, dicite discipulis eius et Petro, quia praecedit vos in Galileam.

30 Vgl. dazu Evers u.a.: Melodien, Bd. 2/1, S. $153 \mathrm{f}$.

31 Vgl. dazu die Hinweise bei Klesatschke, S. 409, 531. 
Lied versifiziert, nennt gleich im ersten Stollen (V.1-4) den liturgischen Ort dieser Lesung:

\author{
Man list im puch der weisheit here \\ dy hochwirdig epistel, lautten auf dis fest, \\ als Maria auch eylent was \\ uber das pirg gen Juda schnelligklichen \\ $[\ldots]$
}

Gemeint ist hier die Epistel zum Fest Mariä Heimsuchung (2. Juli). Das Evangelium zu diesem Fest (Lk 1,39-47) versifiziert dann das Lied ${ }^{1} \operatorname{Regb} / 4 / 653$, worauf $\mathrm{zu}$ Beginn der dritten (Schluss-) Strophe hingewiesen wird, die sich der Deutung des versifizierten Textes widmet (V.1 f.: In dissem ewangeliumen / zw mercken sint drey sach, verste, vor vnde nach). ${ }^{32}$ In den vorreformatorischen Bibelversifikationen findet sich die Praxis, neben dem Festtag auch den liturgischen Ort der Perikope zu nennen, (sieht man von Michel Beheim ab) nur bei diesen beiden Liedern und einmal ('Regb/4/557b) in der ,Wiltener Handschrift' (München, BSB, Cgm 5198). ${ }^{33}$

Hans Sachs belässt es hingegen in seinen Bibelversifikationen bei der Erwähnung des Festes, ${ }^{34}$ die er aber auch oft weglässt. Wichtig ist ihm hingegen die durchgängige Quellenangabe der versifizierten Bibelstelle nach dem Modell Mathäus der evangelist ( $\left.{ }^{2} \mathrm{~S} / 111\right)$, Mathäus vns peschreiben ist $\left({ }^{2} \mathrm{~S} / 112\right)$ und vielen weiteren Variationen..$^{35}$ Das Muster dafür konnte Hans Sachs in seinem Berliner Autograph bei den Liedern ${ }^{1} \mathrm{Regb} / 4 / 652$ (I,1: Man list im puch der weisheit here) und ${ }^{1}$ Folz/132 (s. o.; I,15: secundum Mateum, merckt wy) finden.

Zudem boten die Lieder ${ }^{1} \operatorname{Regb} / 4 / 652$ und ${ }^{1} \operatorname{Regb} / 4 / 653$ Orientierung für ein textnahes Versifizieren von Bibelstellen. In ${ }^{1} \mathrm{Regb} / 4 / 652$ wird die lateinische Epistel (Hld 2,8-14) mit geringen Ausnahmen ziemlich vorlagengetreu wieder-

32 Vgl. auch im weiteren Verlauf der Strophe die Berufung auf das Evangelium: als dis ewangely bestet (V. 18).

33 Dort lautet die Überschrift bei ${ }^{1} \operatorname{Regb} / 4 / 557 \mathrm{~b}$ : von vnser lieben frawen das ewangelium das da haisset liber generacionis; bei der Parallelüberlieferung ( $\left.{ }^{1} \mathrm{Regb} / 4 / 557 \mathrm{a}\right)$ in der ,Kolmarer Liederhandschrift" (München, BSB, Cgm 4797) wird dagegen allgemeiner auf das Fest und auf den Titel der Perikope (zu diesem Fest) verwiesen: von vnser frauwen geburt liber generacionis.

34 Beispiele aus dem Jahr 1526 (Beginn seiner Bibelversifikationen) sind ${ }^{2} \mathrm{~S} / 131$ (der weinacht par versifiziert mit Lk 2,1-20 das Weihnachtsevangelium) und ${ }^{2} \mathrm{~S} / 132$ (weinacht par auf new jar und liechtmes mit Lk 2,21-40, dem Bericht von der Darstellung Jesu).

35 So noch in der letzten Bibelversifikation ( $\left.{ }^{2} \mathrm{~S} / 5426 \mathrm{a}\right)$, die auf den 14. Oktober 1560 datiert ist: Im andren dail der Cronica 
gegeben. Als Beispiel sei der zweite Stollen der ersten Strophe zitiert, mit dem die Versifikation einsetzt (V. 5-8): ${ }^{36}$

Sich an, er kumpt und pringet ere,

uber hüpffet die perg und dal mit seiner glest.

gleich einem rechgeis er sich mas

und gleich ein hirsslein jungck kam er gestrichen.

Bei der Versifikation in ${ }^{1}$ Regb/4/653 meldet sich der Autor mit kleinen Zusätzen etwas häufiger zu Wort. Besonders deutlich ist das bei der Wiedergabe von Lk 1,41a (Et factum est, ut audivit salutationem Mariae Elisabeth, exultavit infans in utero eius) im zweiten Stollen der ersten Strophe (V. 5-8):

Elysabet gros freud entpfinge,

die frucht in irem leib knyet, den hern verja

frolockent, mit kintlicher fle

es seinem schopffer da beweisset ere.

In beiden Liedern schließen sich an die Versifikation ganz in spätmittelalterlicher Tradition deutende und erläuternde Autortexte an, die jedoch deutlich erkennbar vom versifizierten Bibeltext abgesetzt sind. Die Trennungslinie entspricht jeweils der Strophengrenze: Bei ${ }^{1} \mathrm{Regb} / 4 / 652$ beginnt der durchgehende Autortext in der zweiten, bei ${ }^{1} \mathrm{Regb} / 4 / 653$ in der dritten Strophe. Dazu treten sprachliche Markierungen, mit denen ein Rückverweis auf die versifizierte Perikope erfolgt ( ${ }^{1} \mathrm{Regb} / 4$ / 652: Disser epistel wort; ${ }^{1}$ Regb/4/653: In dissem ewangeliumen). Nach diesem separierenden Signal meldet sich unüberhörbar der Autor zu Wort. Bei ${ }^{1}$ Regb/4/652 unterrichtet er im ersten Stollen der zweiten Strophe darüber, dass die epistel zur Zeit der schwangeren Maria das ewangelium gewesen sei (V. 1-4):

Disser epistel wort sein gründe vrsprüncklich das ewangelium von der zeit, als Maria noch tragen tet

den himel her, der zw wir kam gesprüngen.

In ${ }^{1} \mathrm{Regb} / 4 / 653$ wird zu Beginn der dritten Strophe erläutert, dass vom Gruß des Engels in dieser Perikope das Gebet Ave Maria abgeleitet ist (V. 1-4):

36 Vgl. dazu Hld 2,8f.: ${ }^{8}$ (Vox dilecti mei!) Ecce iste venit saliens in montibus transiliens colles. ${ }^{9}$ Similis est dilectus meus capreae hinnuloque cervorum. 
In dissem ewangeliumen

zw mercken sint drey sach, verste, vor vnde nach,

dar durch das pet der englisch grus

gesamelt wart nach cristenlichem orden.

Bei den Deutungen und Erläuterungen folgen die Autortexte unterschiedlichen Wegen. Nach der ,geschichtlichen' Verortung der Bibelstelle folgt in ${ }^{1}$ Regb/4/652 in allegorischer Weise ${ }^{37}$ eine heilsgeschichtliche Erklärung der Bibelstelle, wobei der Bogen von der Inkarnation bis zur Auferstehung Jesu gespannt wird. Zur Illustration des Verfahrens sei der erste Stollen der dritten Strophe zitiert (V. 1-4):

Die zeit der reben zw beschneiden

hy pey das leiden Cristi clerlichen verstett,

dar zw fünf mal sein plut vergos.

das erst in der beschneidung sich vorfinge.

Ein anderes Ziel verfolgen hingegen die Erläuterungen im Lied ${ }^{1}$ Regb/4/653. Sie wollen die biblischen Quellen und die Herkunft des Gebetes Ave Maria erläutern. Deswegen setzt die zweite Strophe die Versifikation des Magnifikat (Lk 1,48-55) fort, mit dessen Beginn die Versifikation des Festtagsevangeliums (Lk 1,39-47) in der ersten Strophe schließt. Diese Erweiterung hat jedoch eine eigene Funktion: Sie versteht sich als Gebet. Entsprechend endet die Versifikation mit der Doxologie als Gebetschluss und mit einem Hinweis auf Maria als Urheberin des Gebets und dessen Wirkung für die Betenden (V. 20-23):

er sey dem vatter, sun vnd geist,

den drey persan in einiger gotheit.

vnd disse wort Maria ret,

durch die got alles vbel von vns scheit.

Die dritte Strophe weist im ersten Stollen auf die biblische Grundlage des Ave Maria hin, dann auf die Komposition des Gebets aus den beiden versifizierten Bibelstellen (V. 2: vor vnde nach) und auf den christlichen Ursprung dieser Ver-

37 Eine detaillierte allegorische Deutung der Opferung Isaaks (Gen 22,1-19) gibt Hans Sachs 1528 in seinem Lied ${ }^{2} \mathrm{~S} / 221$. Auch sonst kennt Sachs als Anhänger der Reformation die Allegorese bei versifizierten Bibelstellen. So 1527 die Lieder ${ }^{2}$ S/172 (Erschaffung der Eva [Versifikation der vier Verse Gen 2,21-24] als Präfiguration für die Erlösungstat Jesu und für die nachfolgende Heilsgeschichte) und ${ }^{2} \mathrm{~S} / 162$ (Die cluckhenn: Versifikation des Verses Mt 23,37, dessen Wendung quemadmodum gallina congregat pullos suos sub alas auf Christus gedeutet wird). 
knüpfung (V. 1-4; s.o.). Ergänzend wird am Schluss des Abgesangs noch auf Ergänzungen durch die Kirche ${ }^{38}$ hingewiesen (V. 19-23):

darnach dy kirch das pet vollent

vnd seczt dar zw die wort Jesus Cristus.

20

den sprechen wir, Amen dar zW

meit won vns pey mit deinem sun Jesus.

Der zweite Stollen leitet dann zum Ave Maria selbst über, dann folgt im Abgesang der lateinische Text des Gebets, das in Form eines Glossenliedes in deutscher Sprache kommentiert wird (V. 9-18):

Er [der Engel] grüst dich: ave Maria, sprach: gracia plena, dominus tecum.

die cristlich kyrch vergicht vns da

das wort: Maria, propter hoc sis mecum.

das ander: benedicta tu

in mulieribus.

ir mum gen ir mit worten hie beschlus,

da sie mit lauter stim schrey: et

benedictus fructus tui ventris,

als dis ewangely bestet.

Die Autortexte im Anschluss an die Versifikationen bei beiden Liedern unterscheiden sich deutlich in ihrer Thematik, aber sie treffen sich in dem Bemühen um eine pragmatische Zielsetzung ohne spekulative religiöse oder dogmatische Ausgriffe. In ${ }^{1}$ Regb/4/652 ist die Deutung im Blick auf das kirchliche Fest homiletisch ausgerichtet und verdeutlicht seine heilsgeschichtliche Bedeutung. In ${ }^{1}$ Regb/4/653 sind die Erläuterungen eher einem katechetischen Anliegen verpflichtet. Neben der Textnähe bei der Versifikation konnte auch diese Form der deutenden Autortexte in beiden Liedern Hans Sachs Anregungen für seine eigenen Bibelversifikationen geben.

Fasst man die Beobachtungen zu den zehn Liedern im Berliner Hans SachsAutograph Berlin, SBB, mgq 414 zusammen, dann vermitteln sie ein ziemlich buntes Bild, dessen Uneinheitlichkeit neben dem numerischen Argument ein weiteres Indiz dafür liefert, dass die Bibelversifikation vor der Reformation kein fester Liedtyp war. ${ }^{39}$ Das bestätigt auch die ,Kolmarer Liederhandschrift', die äl-

38 Vgl. auch III,11f.: die cristlich kyrch vergicht vns da / das wort: Maria, propter hoc sis mecum. 39 Vgl. dazu Anm. 5. 
teste und neben dem Berliner Autograph mit sieben Liedern die umfangreichste vorreformatorische Sammlung mit Bibelversifikationen. ${ }^{40}$ Trotz dieser Diversität bei letztlich wenigen Beispielen konnte Hans Sachs für seine Grundlegung der textnahen Bibelversifikation als zentralem Liedtyp des Meistergesangs in der Reformationszeit wichtige Einsichten und Anregungen von den vorreformatorischen Belegen in seiner autographen Sammlung erhalten.

Die vielleicht wichtigste Erkenntnis war wohl, dass die Versifikation von Bibelstellen in der Form von Meisterliedern überhaupt möglich ist. Das mag nach der Flut der Bibelversifikationen in der Reformationszeit einem heutigen Betrachter als eine Selbstverständlichkeit erscheinen, aber die geringe Zahl vorreformatorischer liedhafter Bibelversifikationen scheint auf eine Scheu vor poetischen Bearbeitungen des kanonischen (teilweise sogar liturgisch verorteten) Textes in der Form von Sangsprüchen und Meisterliedern zu deuten. Nur vereinzelt ließen sich unbekannte und bekannte Autoren von solchen Vorbehalten nicht abhalten. Mit ihren Versuchen stellten sie unter Beweis, dass nicht nur Paraphrasen, sondern sogar textnahe Bibelversifikationen in Liedform möglich sind. Auf dieser Einsicht konnte Hans Sachs dann aufbauen, als er nach der Reformation in einem theologisch und religiös neuen Kontext den Liedtyp der textnahen Bibelversifikation als eine laikale Form der biblischen Wortverkündigung schuf.

Der Anspruch auf Textnähe stand vor der Reformation freilich nicht im Mittelpunkt der Bibelversifikationen. ${ }^{41}$ Davon scheint das Sachs-Autograph abzuweichen, bei dem vier der zehn Aufzeichnungen (= 40\%) in unterschiedlicher Weise auf Textnähe zielen. ${ }^{42}$ Selbst wenn bei der geringen Zahl von Belegen größte Vorsicht über den Aussagewert solcher Berechnungen angebracht ist und wenn dabei auch der pure Zufall in Rechnung gestellt werden muss, ${ }^{43}$ kannte Sachs nachweisbar vier Belege aus vorreformatorischer Zeit für textnahe Bibelversifikationen, die ihm Ermunterung und Hilfe für sein eigenes Vorhaben sein konnten.

40 Beide Sammlungen decken mit 17 Bibelversifikationen den größten Teil (ausgenommen Michel Beheim) der 23 vorreformatorischen Lieder ab. $\mathrm{Zu}$ den Versifikationen in der ,Kolmarer Liederhandschrift‘ vgl. Janota: Bibelversifikationen (im Druck).

41 In der ,Kolmarer Liederhandschrift‘ sind zwei von sieben Versifikationen als textnahe einzustufen (= 28,6\%). Bei dieser Zählung blieb allerdings der ,Hort‘ Peters von Reichbach unberücksichtigt, weil hier ein Leich und kein Sangspruch vorliegt.

42 Es handelt sich um die Lieder ${ }^{1}$ Folz/33b und 132, ${ }^{1}$ Regb/652 und 653.

43 Etwa bei den Liedern ${ }^{1} \mathrm{Regb} / 652$ und 653, die inhaltlich zusammengehören und die beide im Langen Ton Regenbogens stehen. Fasste man sie als eine Einheit, dann reduzierte sich der Anteil der textnahen Versifikationen von 40\% auf 30\%. Dieser Wert entspräche in etwa den $28,6 \%$ in der ,Kolmarer Liederhandschrift‘ (vgl. Anm. 41). 
Nicht weniger aufschluss- und lehrreich mochte für Hans Sachs der Einblick in die spätmittelalterliche Praxis gewesen sein, die Versifikationen variantenreich mit Autortexten zu verknüpfen. Er hat - soweit erkennbar ${ }^{44}$ - diese Tradition teilweise aufgegriffen und sie seinen von der Reformation bestimmten Zwecken angepasst. Kein Vorbild für die Bibelversifikation konnte ihm dabei das Glossenlied sein, wie er es in diesem Zusammenhang (Prolog des Johannes-Evangeliums) aus den Liedern ${ }^{1}$ Marn/7/572 und ${ }^{1} \operatorname{Regb} / 4 / 651$ kannte und das er als Liedform für andere Themen auch selbst verwendete. ${ }^{45}$ Die Mischung aus Bibeltext und kommentierendem Autortext widersprach seiner Vorstellung von einer textnahen Bibelversifikation, die er - vom reformatorischen sola scriptura-Prinzip geleitet - in seinen eigenen Versifikationen praktizierte. Dem reformatorischen Verständnis widersprach es auch, nach Martin Luthers Bibelübersetzung den Bibeltext wie in vorreformatorischer Zeit auf Latein zu zitieren, das den Laien durch eine glossierende Übersetzung erschlossen werden musste. Problematisch war es für Hans Sachs aber auch, den deutschsprachigen Bibeltext kontinuierlich mit Erläuterungen zu durchsetzen, wie es sein Lehrer Lienhard Nunnenbeck ebenfalls am Johannes-Prolog demonstrierte ( ${ }^{1} \mathrm{Nun} / 20$; vgl. auch ${ }^{1} \mathrm{NachtK} / 8 / 1$ ). Und ebenso ungangbar war die raffende Paraphrase ('Nun/26), vor allem, wenn sie so angelegt war, dass sie nur eine biblische Grundlage für eine theologische Thematik lieferte ( ${ }^{1} \mathrm{Frau} / 23 / 6 \mathrm{~b}$ : würdiger Empfang der Eucharistie).

Anschließen konnte Hans Sachs bei seinen Versifikationen hingegen bei den Beispielen, die sich um eine textnahe Bibelversifikation bemühten und dabei Bibel- und Autortext deutlich erkennbar voneinander trennten. Das gilt zunächst für die Lieder ${ }^{1}$ Folz/33 und ${ }^{1}$ Folz/132. Deren Struktur konnten als Muster jedoch nicht unverändert übernommen werden, wenn der Blick zuerst und unverstellt auf den Bibeltext gerichtet sein sollte, so wie das den reformatorischen Vorstellungen entsprach. Das war bei ${ }^{1}$ Folz/33 nicht der Fall, weil die textnahe Versifikation auf die einzelnen Liedstrophen aufgeteilt und strophenweise kommentiert wurde. ${ }^{46}$ Bei ${ }^{1}$ Folz/132 wiederum war der Versifikation eine Präfiguration der Auferstehung

44 Da genauere Untersuchungen zu den zahlreichen meist unedierten Bibelversifikationen von Hans Sachs fehlen, stehen die nachfolgenden Ausführungen unter Vorbehalt. Sie beruhen zum größten Teil auf den Liedregesten im RSM, die ich für die Jahre 1526-1528 und 1558-1560 durchgesehen habe. Um wenigstens einen kleinen Teil dieser überbordenden Fülle zugänglich zu machen, arbeite ich an einer Studie über die ca. 60 Versifikation der Weihnachtsgeschichte (Lk 2,1-20) in den Meisterliedern der Reformationszeit.

45 Vgl. dazu die Hinweise in Anm. 13.

46 Im Jahre 1528 findet sich dieses Muster allerdings auch bei Hans Sachs in zwei Liedern: ${ }^{2} \mathrm{~S} / 211$ (abschnittsweise Auslegung von Psalm 23) und in ${ }^{2} \mathrm{~S} / 224$ (versweise Auslegung von Psalm 127). Vielleicht nehmen die Psalm-Versifikationen eine Sonderstellung ein; vgl. dazu auch Anm. 47. 
Jesu als Autortext vorgeschaltet. ${ }^{47}$ Eine akzeptable Alternative war die Abfolge von Bibel- und deutlich davon getrenntem Autortext wie in den Liedern ${ }^{1}$ Regb/4/652 und ${ }^{1} \operatorname{Regb} / 4 / 653$.

Tatsächlich übernahm Hans Sachs im ersten Jahr seiner eigenen Bibelversifikationen (1526) diese Struktur: Bei acht der insgesamt 16 Versifikationen schließt sich an den Bibel- ein erläuternder und deutender Autortext an. Bei sechs weiteren Liedern reduziert sich der Autortext auf wenige Schlussverse. ${ }^{48}$ Dabei ist die Grenze allerdings im Blick auf den Umfang und die thematische Ausrichtung zum erläuternden und deutenden Autortext fließend. Deswegen empfiehlt es sich wohl, die Kombination von Versifikation und Schlussversen nicht als eigenen Typ anzusetzen, sondern in ihm eine Variante der Verbindung von Versifikation und erklärend-deutendem Autortext zu sehen. Die Abgrenzung dieser Variante scheint mir bei Hans Sachs nicht unwichtig zu sein, weil ihr reduzierter Autortext einer Tendenz zur bloßen Versifikation einer Bibelstelle folgt. Sie stellt gegenüber der vorreformatorischen Überlieferung ein Novum dar, das sich dem reformatorischen sola scriptura-Ideal verdankt. Mit der Neuheit dieses Versifikationstyps korrespondiert vorerst eine unverkennbare Zurückhaltung gegenüber einer solchen Neuerung: Sachs legt 1526 lediglich zwei Versifikationen ohne Autortext vor. Das ändert sich im Folgejahr 1527: Hier steigt dieser Typ auf 14 Lieder an, was bei insgesamt 49 Versifikationen in diesem Jahr einem Anteil von 28,6\% entspricht. Naheliegend ist daher die Erwartung, dass dieser neue, reformatorische Typ der Bibelversifikation nach und nach bei Hans Sachs die Oberhand gewinnt. Diese Vermutung wird jedoch enttäuscht, wenn man die 71 Bibelversifikationen des Jahres 1528 anschaut, von denen nur neun Lieder $(=12,8 \%)$ frei von einem zusätzlichen Autortext sind. Einen ähnlichen Befund gibt es für die späten Jahre, in denen die Bibelversifikationen bei Hans Sachs deutlich zurückgehen. 1558 liegt

47 Einen einleitenden Autortext mit Verständnishinweisen findet sich bei Hans Sachs aber mehrfach in Psalm-Versifikationen: im Jahr 1527 bei den Liedern ${ }^{2} \mathrm{~S} / 138$ (Psalm 72) und ${ }^{2} \mathrm{~S} / 170$ (Psalm 104), im Jahr 1528 bei den Liedern ${ }^{2} \mathrm{~S} / 218$ (Psalm 25) und ${ }^{2} \mathrm{~S} / 250$ (Psalm 10). Daneben hat Sachs von Anfang an auch Psalm-Versifikationen ohne einen solchen Vorspann: im Jahr 1526 bei den Liedern ${ }^{2} \mathrm{~S} / 113$ (Psalm 33), ${ }^{2} \mathrm{~S} / 121$ (Psalm 103) und ${ }^{2} \mathrm{~S} / 129$ (Psalm 115), im Jahr 1527 beim Lied ${ }^{2} \mathrm{~S} /$ 166 (Psalm 22) und im Jahr 1528 bei den Liedern ${ }^{2} \mathrm{~S} / 208$ (Psalm 148), ${ }^{2} \mathrm{~S} / 209$ (Psalm 34), ${ }^{2} \mathrm{~S} / 219$ (Psalm 2), ${ }^{2} \mathrm{~S} / 226$ (Psalm 92), ${ }^{2} \mathrm{~S} / 240$ (Psalm 94), ${ }^{2} \mathrm{~S} / 241$ (Psalm 133) und ${ }^{2} \mathrm{~S} / 281$ (Psalm 98); im Jahr 1558 beim Lied ${ }^{2} \mathrm{~S} / 5128$ (Psalm 118). Über die Rolle des Psalmliedes als reformatorisches Kirchenlied außerhalb des Meistergesangs vgl. Anm. 3. - Zu allegorischen Deutungen bei Versifikationen von Hans Sachs vgl. die Hinweise in Anm. 37.

48 Als Beispiel sei aus diesem Jahr die Versifikation von Lk 2,1-20 (Geburt Jesu) in dem dreistrophigen Lied ${ }^{2} \mathrm{~S} / 131$ genannt. Hier endet die Schlussstrophe mit der Aufforderung: ein ider Cristen mensch lob sprech, / frolock vnd jubelire, / hoffire / Jesu Cristo, durch den wir all erlösset sint (V. 20-23). 
das Verhältnis bei 18:3 (= 16,7\%), 1559 bei 16:4 (= 25\%) und in den Jahren 1560 1567 bei 5:1.

Dieser Befund ist bemerkenswert, weil Hans Sachs nach seinem Bekenntnis zur Reformation mit seinen textnahen Bibelversifikationen nicht nur einen neuen Liedtyp innerhalb des Meistergesangs etabliert, sondern mit den reinen Versifikationen ohne erklärende und deutende Autortexte im Vergleich mit der spätmittelalterlichen Überlieferung ein Novum geschaffen hat. Dennoch stand diese auffällige Neuerung bei Hans Sachs nicht im Mittelpunkt seiner zahlreichen Bibelversifikationen. Vielmehr setzte er hauptsächlich die vorreformatorische Tradition fort, in der versifizierter Bibel- und Autortext eine Einheit bildeten. Neu ist allerdings dabei, dass Sachs im Unterschied zur früher nicht selten unbekümmerten Vermengung der beiden Textebenen - wohl als Reflex seiner reformatorischen Einstellung - auf deren deutliche Trennung achtete. Auf diese Weise überlappen sich bei Hans Sachs Tradition und Innovation, sein Festhalten an einem als gut befundenen Erbe und sein Mut zu Neuerungen, die den Meistergesang aus seiner Erstarrung befreiten und damit zugleich den Grundstein für eine neue Tradition legten.

Eine nicht unwichtige Rolle bei diesem Entwicklungsgang scheint für Hans Sachs sein Berliner Autograph mgq 414 gespielt zu haben. Die vergleichsweise große Zahl von Bibelversifikationen in dieser vorreformatorischen Sammlung mochte Sachs die Anregung gegeben haben, die Bibelversifikation als Meisterlied in den Dienst der Reformation zu stellen. Die insgesamt zehn Aufzeichnungen boten ihm aber auch hinlänglich Anschauungsmaterial dafür, welche Formen der Versifikation sich im reformatorischen Sinn für die Weitergabe von Bibelabschnitten von Laien für Laien im Rahmen des Meistergesangs eigneten und auch weiterentwickeln ließen. Bei der konservativen Ausrichtung der Meistersinger war es offenkundig angebracht, die Bibelversifikation als neuen Liedtyp breit in herkömmliche Muster der Versifikation einzubetten. Dafür besaß Hans Sachs in seiner ersten autographen Sammlung der ihm zugänglichen und von ihm aus formalen wie inhaltlich Gründen geschätzten Überlieferung eine Art von Rückversicherung. Sammlungen dieser Art waren immer rückwärtsgewandt, aber gerade dadurch eröffneten und legitimierten sie die Möglichkeit für Neues. Auch das zeigen die Bibelversifikationen im Berliner Autograph mgq 414, wenn man sie mit Blick auf die eigenen Versifikationen betrachtet, die Hans Sachs später im Geiste der Reformation verfasste. 


\section{Literaturverzeichnis}

Art. ,Plenarien [Korr./Nachtr.]. In: VL² 11 (2004), Sp. 1249.

Baldzuhn, Michael: Vom Sangspruch zum Meisterlied. Untersuchungen zu einem literarischen Traditionszusammenhang auf der Grundlage der Kolmarer Liederhandschrift. Tübingen 2002 (MTU 120).

Brunner, Horst: ...das Reich mus vns doch bleiben. Einführung in Luthers Kirchenlieder. In: Blick. Magazin der Universität Würzburg. Heft 1/1998, S. 140-146.

Brunner, Horst: Annäherungen. Studien zur deutschen Literatur des Mittelalters und der Frühen Neuzeit. Berlin 2008 (Philologische Studien und Quellen 210).

Brunner, Horst: Luther und das deutsche Kirchenlied im 16. Jahrhundert. In: Reformation und katholische Reform zwischen Kontinuität und Innovation. Hg. von Fran Kleinehagenbrock u.a. Würzburg 2019 (Publikationen aus dem Kolleg „Mittelalter und Frühe Neuzeit 6), S. $249-265$.

Brunner, Horst: Meistergesang und Reformation. Die Meistergesangbücher 1 und 2 des Hans Sachs. In: Literatur und Laienbildung im Spätmittelalter und in der Reformationszeit. Symposion Wolfenbüttel 1981. Hg. von Ludger Grenzmann, Karl Stackmann. Stuttgart 1984 (Germanistische Symposien. Berichtsbände 5), S. 732-742; revidierter Wiederabdruck in Brunner, Horst: Literarisches Leben. Studien zur deutschen Literatur. Berlin 2018 (Philologische Studien und Quellen 266), S. 276-290.

Cramer, Thomas: Die kleineren Liederdichter des 14. und 15. Jahrhunderts. Bd. 4. München 1985.

Evers, Ute, Johannes Janota (Hgg.): Die Melodien der lateinischen Osterfeiern. Editionen und Kommentare. 4 Teilbände. Berlin, Boston 2013.

Janota, Johannes: Die Bibelversifikationen in der Kolmarer Liederhandschrift. In: Die Kolmarer Liederhandschrift und ihr Umfeld (3.-4. September 2018) (im Druck).

Klesatschke, Eva: Lienhard Nunnenbeck. Die Meisterlieder und der Spruch. Edition und Untersuchungen. Göppingen 1984.

Mayer, August L. (Hg.): Die Meisterlieder des Hans Folz. Aus der Münchener Originalhandschrift und der Weimarer Handschrift Q. 566 mit Ergänzungen aus anderen Quellen. Berlin 1908 (DTM 12).

Reinitzer, Heimo, Olaf Schwencke: Art. ,Plenarien‘. In: VL² 7 (1989), Sp. 737-763.

Wachinger, Burghart: Michel Beheim. Prosabuchquellen - Liedvortrag - Buchüberlieferung. In: Poesie und Gebrauchsliteratur im deutschen Mittelalter. Würzburger Kolloquium 1978. Hg. von Volker Honemann u. a.Tübingen 1979.

Wachinger, Burghart: Lieder und Liederbücher. Gesammelte Aufsätze zur mittelhochdeutschen Lyrik. Berlin, New York 2011. 